\title{
Characterization of Sulfonated Polysulfone Polymers by EELS
}

\author{
Chen Wang ${ }^{1}$, Stephen J. Paddison ${ }^{2}$, John R. Dunlap ${ }^{3}$, and Gerd Duscher ${ }^{4}$ \\ 1. Division of Applied Research and Technology, National Institute for Occupational Safety and Health, \\ Cincinnati, $\mathrm{OH}, 45226$, USA \\ 2. Department of Chemical and Biomolecular Engineering, University of Tennessee, Knoxville, TN \\ 37996, USA \\ 3. Advanced Microscopy and Imaging Center, University of Tennessee, Knoxville, TN 37996, USA \\ 4. Department of Materials Science and Engineering, University of Tennessee, Knoxville, TN, 37996, \\ USA
}

A novel class of polysulfone ionomers have recently been synthesized and proposed as candidate electrolyte membrane materials for fuel cells.[1] These materials show superior proton conductivity and thermal stability due to their unique backbone structures consisting of sulfonated aromatic rings and sulfone units $\left(-\mathrm{SO}_{2}-\right)$. The local hydration and proton conductivity are closely related to the degree of backbone sulfonation and the spacing of the sulfone units. Electron energy-loss spectroscopy (EELS) has been undertaken to understand the conformational changes in the backbone of various perfluorosulfonic acid (PFSA) ionomers.[2] The combination of spectroscopy and simulation has successfully revealed the conformational dependence of the EEL spectra for PFSA ionomers. In the present work, the specific features in the low-loss and core-loss spectra of light elements (e.g., C and S) were investigated to understand the backbone chemistry of polysulfone ionomers.

We have performed EELS with a $200 \mathrm{kV}$ Zeiss Libra 200 TEM/STEM equipped with a monochromator to investigate the spectral characteristics of three types of sulfur-containing aromatic polymers: poly(1,4-phenylene ether-ether-sulfone) (PEES); poly-(phenylene sulfide) (PPS) and sulfonated poly(phenylene sulfone) (sPSO $)_{2}$, as seen in Fig.1. Both low-loss EEL spectra and the energy-loss near-edge structure (ELNES) have been acquired with a high energy resolution of $0.15 \mathrm{eV}$. The thin sections of the samples were prepared by cryo-microtome and examined in the cryo environment to minimize sample damage due to electron beam exposure.

The spectral dependence of the different aromatic backbone structures were investigated by low-loss spectra, C K-edges and S- $\mathrm{L}_{2,3}$ edges as shown in Fig. 2. The spectra show distinct features in the lowloss region and the onset of the $\mathrm{C}$ K-edges for PEES, PPS, and $\mathrm{sPSO}_{2}$. The addition of sulfonic acid groups $\left(-\mathrm{SO}_{3} \mathrm{H}\right)$ or ether linkages (-O-) in backbones can both alter the intensities and shapes of $\pi$ peaks in $\mathrm{C}$ K-edges and near edge structures. A strong singlet peak in the S-L2,3 ELNES was observed in $\mathrm{sPSO}_{2}$ ionomers, which can be used to characterize the sulfonate groups directly attached to the aromatic backbones. The fine structures of the S-L2,3 ELNES of PPS and PEES were also identified and possessed features similar to the previously reported X-ray absorption spectra of sodium sulfate.[3]

\section{References:}

[1] M. Schuster et al., Macromolecules, 42 (2009), 3129-3137.

[2] C. Wang et al., RSC Adv., 5 (2015), 2368-2373.

[3] F. Jalilehvand, Chem. Soc. Rev., 35 (2006), 1256-1268. 


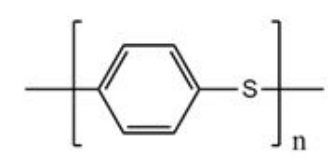

PPS

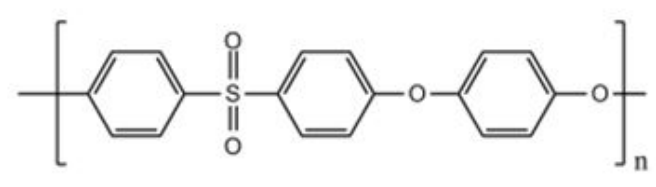

PEES

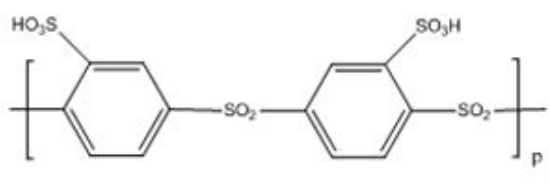

$\mathrm{sPSO}_{2}$

Figure 1. Chemical structures of repeat units of poly-(1,4-phenylene ether-ether-sulfone) (PEES); poly(phenylene sulfide) (PPS) and sulfonated poly-(phenylene sulfone) $\left(\mathrm{sPSO}_{2}\right)$.
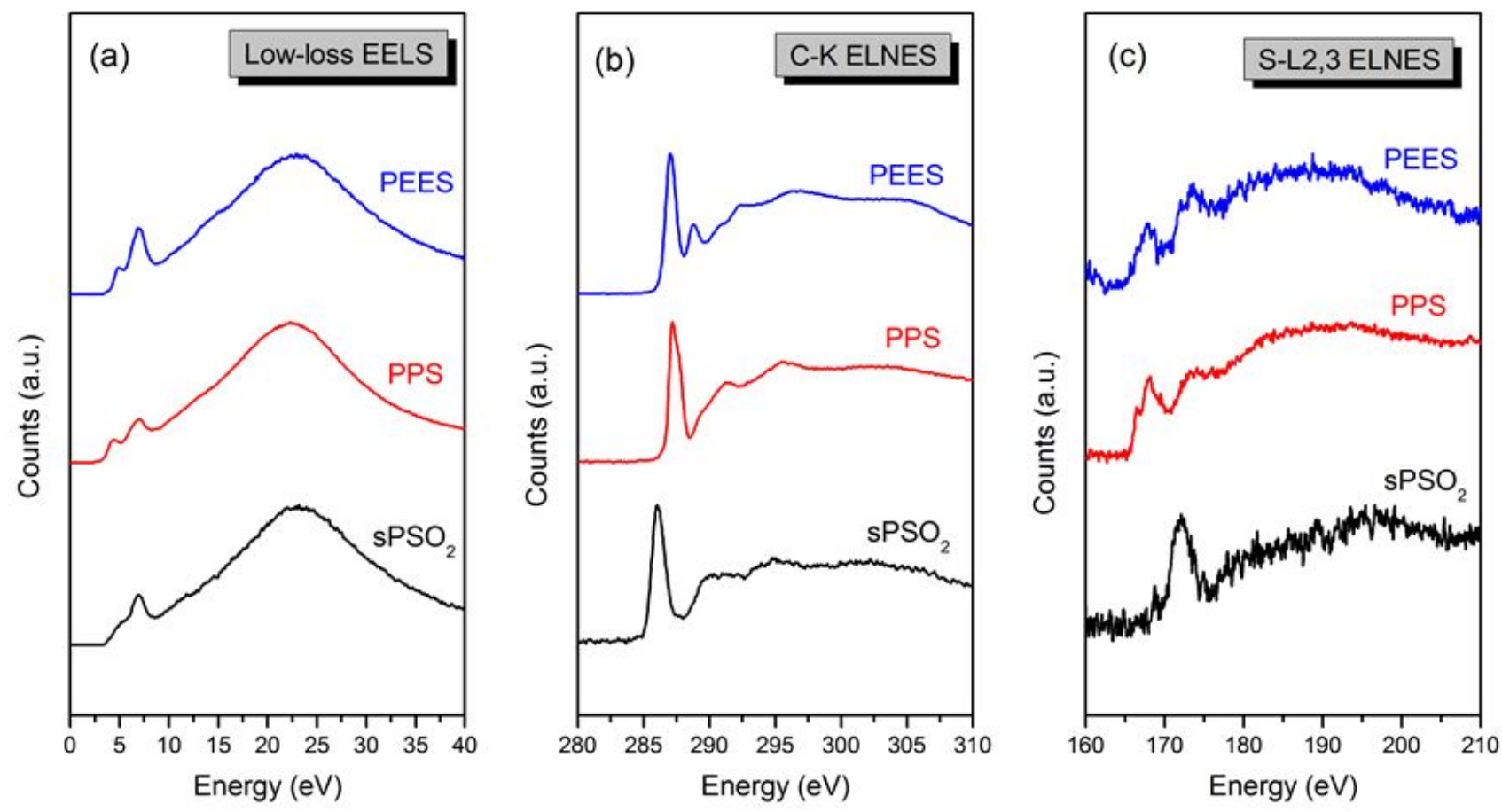

Figure 2. Measured EEL spectra of PEES, PPS, and $\mathrm{SPSO}_{2}$ : (a) low-loss (0-40 eV) after the removal of zero-loss (logarithmic function) and plural scattering (Fourier-log deconvolution); (b) C-K ELNES (280-310 eV) and (c) S-L2,3 ELNES (160-210 eV) after background subtractions (power-law function) and single scattered removal (Fourier-ratio deconvolution). 